\title{
Extracellular vesicles in chronic obstructive pulmonary disease (COPD)
}

\author{
Hannah E. O'Farrell ${ }^{1}$, Ian A. Yang ${ }^{1,2}$ \\ ${ }^{1}$ UQ Thoracic Research Centre, Faculty of Medicine, The University of Queensland, Brisbane, Australia; ${ }^{2}$ Thoracic Program, The Prince Charles \\ Hospital, Metro North Hospital and Health Service, Brisbane, Australia \\ Contributions: (I) Conception and design: All authors; (II) Administrative support: None; (III) Provision of study materials or patients: None; (IV) \\ Collection and assembly of data: None; (V) Data analysis and interpretation: None; (VI) Manuscript writing: All authors; (VII) Final approval of \\ manuscript: All authors. \\ Correspondence to: Hannah E. O’Farrell. UQ Thoracic Research Centre, Room 2, Level 1 Clinical Sciences Building, The Prince Charles Hospital, \\ Rode Road, Chermside, QLD, Australia. Email: hannah.ofarrell@uq.net.au.
}

\begin{abstract}
Chronic obstructive pulmonary disease (COPD) is a heterogeneous disease characterised by chronic inflammation and significant airflow obstruction that is not fully reversible, and is one of the leading causes of morbidity and mortality worldwide. Extracellular vesicles (EVs) (including apoptotic bodies, microvesicles and exosomes) are small membrane-bound vesicles released by nearly all cell types and can be found in various bodily fluids including blood, sputum and urine. EVs are key mediators in cell-cell communication due to their ability to exchange information to recipient cells, influencing physiological and pathological conditions using their bioactive cargo (DNA, RNA, miRNA, proteins and other metabolites). Therefore the main aim of this review is to highlight recent evidence of the potential use of EVs as diagnostic and therapeutic biomarkers for COPD managements, as well as EVs potential role in COPD pathogenesis. As EVs have been under intense investigation as diagnostic and therapeutic biomarkers for lung disease, in relation to COPD, key studies have identified EVs as potential biomarkers to distinguish exacerbations from stable state, and to characterise COPD phenotypes. EVs are also linked to key inflammatory mediators in COPD progression. In addition, bacteria and their EV cargo influence the lung microenvironment. Further recent therapeutic approaches and advances have seen EVs bioengineered as novel drug delivery vehicles, which could potentially have clinical utility for lung diseases such as COPD.
\end{abstract}

Keywords: Extracellular vesicles (EVs); chronic obstructive pulmonary disease (COPD); biomarkers; therapeutics

Submitted Aug 22, 2019. Accepted for publication Oct 04, 2019.

doi: $10.21037 /$ jtd.2019.10.16

View this article at: http://dx.doi.org/10.21037/jtd.2019.10.16

\section{Introduction}

\section{COPD}

Chronic obstructive pulmonary disease (COPD) is a preventable and treatable respiratory disease, which is characterised by persistent respiratory symptoms with significant obstruction of airflow that is not fully reversible and progressive, with features of chronic airway inflammation in the lungs in response to noxious particles or gases $(1,2)$. Symptoms characteristic of COPD include exertional breathlessness, chronic cough, sputum production and exacerbations of COPD $(1,2)$. COPD is one of the leading causes of morbidity and mortality worldwide, resulting in significant economic and social burden on society (3). In Australia a reported \$929 million AUD (\$629 million USD) per annum is spent on direct health expenditure attributed to COPD, significantly contributing to the health care burden (4). COPD is predicted to become the 3 rd leading cause of death world wide by 2030 unless urgent action is taken to reduce underlying risk factors (5).

Exacerbations of COPD are defined as the worsening of symptoms above a patient's usual baseline, which may require 
pharmacological intervention and or hospitalisation (6). Patients who experience frequent exacerbations in one year will have a higher frequency in following years, considerably impacting on their quality of life (7).

As exacerbations are serious events that accelerates long-term decline in lung function and quality of life, it is important for more research to be undertaken to understand the pathogenesis of COPD exacerbations and underlying risk factors that can be modified through targeted preventive measures and interventions. In addition to developing a better understanding of environmental factors triggering exacerbations, further knowledge is needed about cellular and molecular processes involved in COPD exacerbations and disease progression. An increasingly important field of research relates to extracellular vesicles $(\mathrm{EV})$, which are produced by cells, and will be the focus of this review.

\section{EVs}

\section{Biogenesis}

Production of EVs is a universal feature of nearly all cells (8). These small enclosed vesicles can be detected in a variety of bodily fluids including blood, bronchoalveolar lavage fluid, tissue, urine, sputum and cell culture supernatant (9). EVs play a key role in cell-cell communication and exert their biological effects in a pleiotropic manner. They do this through direct vesicle membrane interaction with recipient cells, or deliver their bioactive cargo through recipient cell internalisation $(10,11)$.

While EVs are all cell derived vesicles enclosed by a lipid bilayer, their biogenesis and secretion mechanisms distinguish them between 3 main classes, which include: apoptotic bodies, microvesicles and exosomes (Figure 1) (11).

Apoptotic bodies are larger in size $(1-4 \mu \mathrm{m})$ and are only formed during programmed cell death (10). Apoptotic bodies can contain non-coding RNAs, DNA fragments and cell organelles, and previous studies have suggested that genetic information can be transferred by the uptake of apoptotic bodies (10). Cell surface markers for apoptotic bodies include TSP and C3b.

Microvesicles (MVs) are smaller than apoptotic bodies, and larger than exosomes (100-200 nm) and are generated by the outward 'budding' of the plasma membrane releasing the nascent $M V$ into the extracellular space membrane (12). MVs are heterogeneous and their function is determined by their bioactive cargo, which is dependent on their cell of origin from which they were shed (13). Once in the extracellular environment MVs cargo can influence the microenvironment through interaction with recipient cells (12). The release of MVs from the plasma membrane involves ARF1, ARF6 (members of the ADP-ribosylation factor small G-protein subfamily) and TSG101 [component of the endosomal-sorting complex required for transport (ESCRT)] (13).

Exosomes are nanosized vesicles $(30-100 \mathrm{~nm})$ and through intracellular endocytic invagination they are formed and released into the multivesicular body (MVB), generating intraluminal vesicles (ILVs) (14). During early to late endosome maturation, the MVB can then fuse with the plasma membrane, this allows exosomes bioactive cargo to be released into the extracellular space $(15,16)$.

Enzyme neutral sphingomyelinase 2 (nSMase2) is essential in the formation of ILVs in the early endosome (14). The endosomal sorting complex required for transport (ESCRT) is required for ILVs to be delivered into MVBs (14). MVBs can then fuse with lysosomes for cargo degradation (ESCRT-dependent mechanism) or fuse with the plasma membrane, which releases the ILVs as exosomes using RAB GTPases (ESCRT-independent mechanism) $(8,17)$. A number of different proteins can distinguish exosomes which include: tetraspanins, membrane transport and fusion proteins, raft proteins, MHC proteins and targeting/ adhesion molecules, which can be found on exosomes surface membrane (8).

\section{Methods for EV isolation}

In order to study them, a number of methods are available for the isolation of EVs, each with their own advantages and shortcomings, which are important to consider in experimental planning. Traditional EV isolation methods utilize EV size and buoyant density through ultracentrifugation, microfiltration and gel filtration (18-20). Additional methods have since emerged based on EV solubility including precipitation with protamine, sodium acetate and polyethylene glycol (21-23). Recently isolation methods have appeared based on $\mathrm{EV}$ population-specific interactions with molecules on the EV surface as well as microfluidic technologies $(20,24)$.

The International Society for Extracellular Vesicles (ISEV) has recommended guidelines for successful isolation and characterisation of EVs (25). It has been recognised that there is no optimal method for EV isolation, with current methods including size exclusion, immunoaffinity or ultracentrifugation. The most suitable method is therefore dependent on the scientific question proposed 


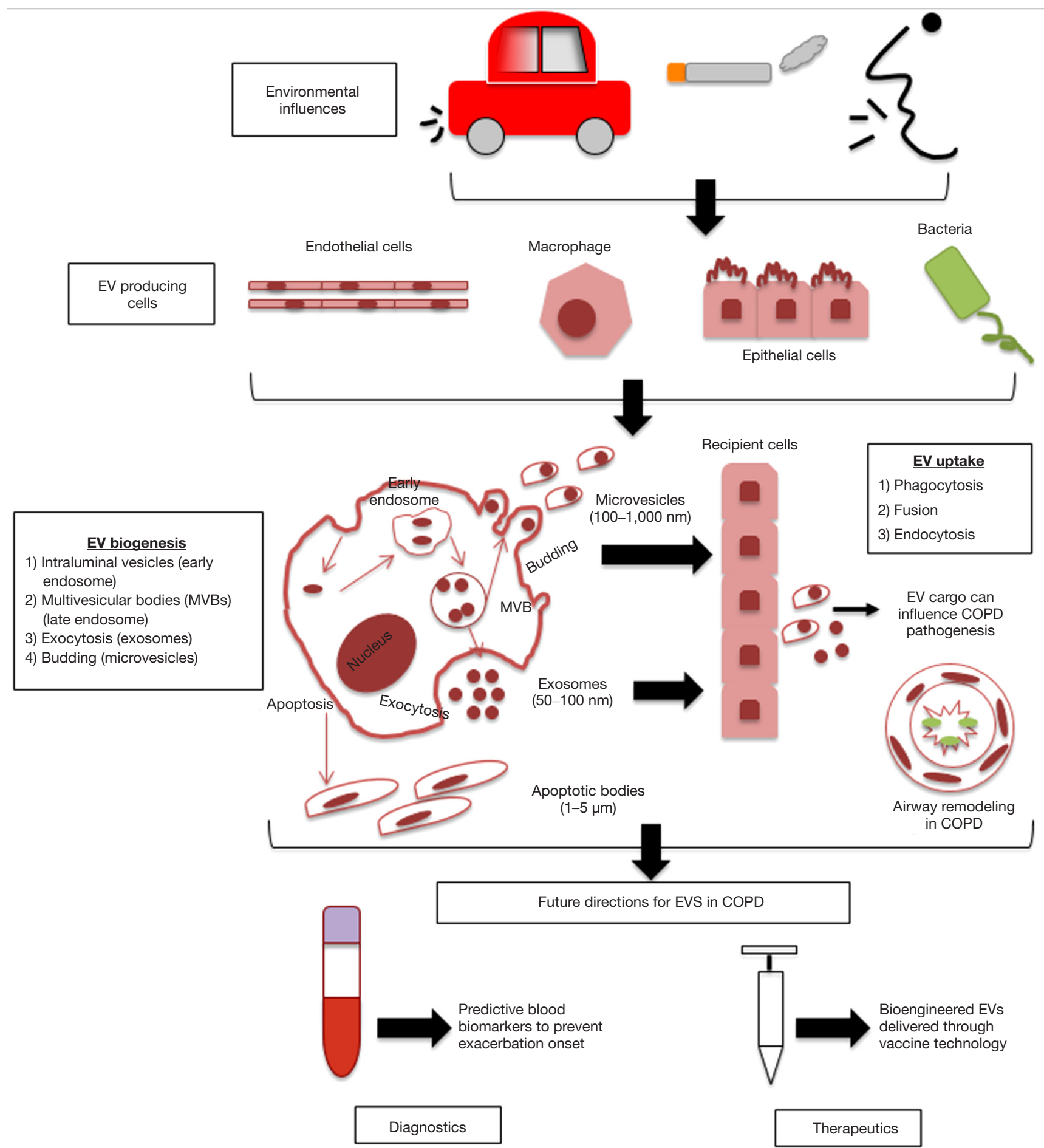

Figure 1 Biogenesis of extracellular vesicles in relation to COPD. Environmental factors including air pollution, noxious particles and bacterial/viral infections can influence the bioactive cargo delivered by EVs. EVs biogenesis begins through the formation of intraluminal vesicles inside MVBs which then bud into early endosomes through to late endosomes. MVBs will then fuse with the plasma membrane, causing the release of content into the extracellular space (exosomes) or through outwards budding and fission with the plasma membrane (microvesicles). Apoptotic bodies are formed during programmed cell death through actin-myosin mediated membrane blebbing. COPD, chronic obstructive pulmonary disease; EV, extracellular vesicle; MVBs, multivesicular bodies. 
and if these methods are applicable with downstream experimental applications, and further that the method chosen is recorded in detail to ensure reproducibility (26). For EV characterization, ISEV has recommended that this is performed at the population and single vesicle level, involving positive and negative protein markers from four categories: (I) EV cytosolic protein, (II) transmembrane EV protein, (III) intracellular but not associated with plasma membrane proteins and (IV) extracellular but not typically associated EV proteins (25). Further to assess population heterogeneity, it is recommended that two complementary technologies are used such as electron microscopy paired with single particle tracking methods (25).

Due to EVs being potent packages of cell-cell communication through the transfer of their bioactive cargo, which reach various sites around the body, further influencing a variety of biological processes of recipient cells, this makes them ideal biomarkers for diagnostics and to investigate novel therapeutics (27), particularly for lung disease (Figure 1) (8). Therefore this review will now discuss how EVs and their cargo could have potential clinical utility as biomarkers and therapeutic agents for COPD disease management and treatment (a list of relevant studies for COPD EVs-some of which will be discussed in further detail below—can be found in Table 1).

\section{The potential role of EVs in COPD pathogenesis}

In normal lung physiology, EVs are likely to play a key role in maintaining homeostasis through intercellular communication in the human airway with studies reporting that lung epithelial cells are the main source of EVs in the lung $(8,45)$. EV composition can be modified through external stimuli, further affecting EV secretion, and the influence EVs have on recipient cells and the surrounding lung microenvironment (8). Such modification may protect against harmful components from smoke exposure, DNA damage and infection $(32,46)$. Studies have previously reported that detrimental immune responses are associated with stress-induced, lung-derived EVs, further contributing to the pathogenesis of chronic inflammatory lung diseases (47). An example is the release of exosomes from activated neutrophils that contain surface-bound neutrophil elastase, which is resistant to degradation by $\alpha 1-$ antitrypsin, leading to excessive alveolar destruction (48).

One of the main causes of exacerbations in COPD patients are bacterial respiratory infections which contribute to disease progression through overproduction of mucus and defective mucociliary clearance in the airways (49). As most cells secrete EVs, when they are released during a pathogen infection they are able to reveal pathogen and host-derived factors, including their composition, origin and state of infection (50). Pathogen EVs play an important role in the regulation of the host's immune response and pathogen uptake and replication (50). In order to promote pathogen survival, replication and pathology, bacterial and viral pathogens can disrupt exosome functions, through their interaction with intracellular transport and signalling pathways, further promoting infection and repressing host pathogen responses $(50,51)$. EVs carrying pathogenderived factors have potential as a diagnostic biomarker (50). A study by Takahashi et al., identified that endothelialderived MVs were significantly increased in stable COPD patients, which further increased in number during an exacerbation, compared to healthy individuals, highlighting the diagnostic potential for MVs to predict patients who are susceptible to exacerbations (29). Another study by Tan et al. assessed the expression levels of plasma exosomes in exacerbating and stable COPD patients and compared to a non-smoking healthy control patients. Results showed that plasma exosomes were significantly increased compared to controls and that exosome expression was associated with plasma CRP, sTNFR1 and IL-6, suggesting that exosomes are involved in the inflammatory process of COPD exacerbations (43).

EVs derived from Gram-negative bacteria [referred in literature as outer membrane vesicles (OMVs)], include molecules such as LPS, adhesion and invasion proteins to host cells, nucleic acids and immunomodulatory factors (52). Recently it was identified that Gram-positive bacteria also produce EVs, which was observed through transmission electron microscopy and proteomic analysis from Staphylococcus aureus and Bacillus subtilis cell culture supernatant (53). In mouse models bacterial-derived EVs have since been shown to induce neutrophilic pulmonary inflammation in a IL-17A dependent manner, and that this neutrophilic pulmonary inflammation which is involved in COPD pathogenesis and induced by E.coli EVs is further abolished when IL-17A is removed $(37,54,55)$. In COPD patients, microbiomes in lung-derived EVs were found to be distinct from microbiomes characterised in lung tissue (42).

Additionally there is evidence to support that respiratory viruses have adapted to use EVs as transporters for viral particles and genomes for transmission and infection (56). In COPD, viruses are a common exacerbation trigger (57). 
Table 1 Key COPD EV studies over the past ten years

\begin{tabular}{lll}
\hline Reference & EV type & Cohort \\
\hline (28) & $\begin{array}{l}\text { Plasma endothelial } \\
\text { microparticles }\end{array}$ & $\begin{array}{l}\mathrm{N}=32 \text { healthy non smokers, } \mathrm{N}=41 \\
\text { healthy smokers with normal spirometry } \\
\text { and } \mathrm{DL}_{\mathrm{co}}, \mathrm{N}=19 \text { healthy smokers with } \\
\text { normal spirometry and low } \mathrm{LL}_{\mathrm{co}}\end{array}$ \\
& $\begin{array}{l}\text { Plasma endothelial } \\
\text { microparticles }\end{array}$ & $\begin{array}{l}\mathrm{N}=80 \text { stable COPD patients, } \mathrm{N}=27 \\
\text { exacerbation COPD patients, } \mathrm{N}=20 \\
\text { healthy non-COPD controls }\end{array}$
\end{tabular}

(30)

Macrophage
microparticles

$$
\text { BALF EVs }
$$

(34)

(35)

$$
\begin{aligned}
& \text { Lung epithelial } \\
& \text { derived EVs }
\end{aligned}
$$

\begin{abstract}
Plasma endothelial microparticles
\end{abstract}

(36)

Primary human bronchial epithelial cells (HBECs) EVs
THP-1 macrophages or hMDMs with or without tobacco smoke extract exposure

$\mathrm{N}=104$ stable COPD patients, $\mathrm{N}=76$ non-COPD patients

Human mononuclear cells exposed to cigarette smoke extract with microparticles isolated

Human bronchial epithelial cells and A549 alveolar cells were then incubated with cigarette smoke extract induced microparticles

$\mathrm{N}=8$ mice $(\mathrm{C} 57 \mathrm{bl} / 6)$ per treatment group, saline control, LPS challenged, air exposed or smoke exposed

BALF and lung tissue were collected

$\mathrm{N}=60$ rats divided into six groups, containing 10 rats each. 3 groups of 10 rats were exposed to cigarettes for 2,4 or 6 months, while the other 3 groups of 10 rats were control groups who were sham-smoked

\section{BEAS-2B human bronchial epithelial cells}

Primary human bronchial epithelial cells

C57BL/6 mice (BALF fluid, lung tissue)

Human lung tissue

BEAS-2B human bronchial epithelial
cells EVs
HBEC EVs

Lung Fibroblasts used to assess EV uptake
Main findings

This study found that smokers with emphysema have increased circulating EMPs, highlighting that monitoring plasma EMP levels would be useful for identifying early emphysema development

This study showed that EMPs CD144+, CD31+ and $\mathrm{CD} 62 \mathrm{E}+$ were significantly elevated in stable COPD patients than healthy controls, further highlighting EMPs biomarker potential for COPD exacerbations

Macrophage-derived EVs attributed to MMP14 were released in response to tobacco smoke extract exposure. Further these EVs may contribute to emphysema in smokers

CD31+ EMPs were significantly elevated in mild COPD and emphysema patients compared to controls, while $\mathrm{CD} 2 \mathrm{E}^{+}$EMPs were significantly elevated in hyperinflation and severe COPD

Macrophage EV release is increased by cigarette smoke extract through the activation of mononuclear cells via intracellular calcium mobilisation. These microparticles contain proinflammatory mediators

The results from this study support that infective agents (bacterial or viral) can trigger EV release in the airways of mice and humans and that ATP drives EVs to release IL- $1 \mathrm{~b}$ and IL-18 through a P2X7/caspase-1-dependent manner

Plasma CD42b-/CD31+ EMPs were significantly increased in rats exposed to cigarette smoke, highlighting their biomarker potential for pulmonary function impairment, which would be useful for evaluating COPD progression

BEAS-2B EVs increased in response to cigarette smoke extract, further inducing RAB27A, further inducing full length fICCN1 enriched EVs

HBEC-derived EVs promoted myofibroblast differentiation in lung fibroblasts in response to cigarette smoke extract. Further miR-210 derived from bronchial epithelial cells targeting ATG7 in lung fibroblasts leads to modulated autophagy

Table 1 (continued) 
Table 1 (continued)

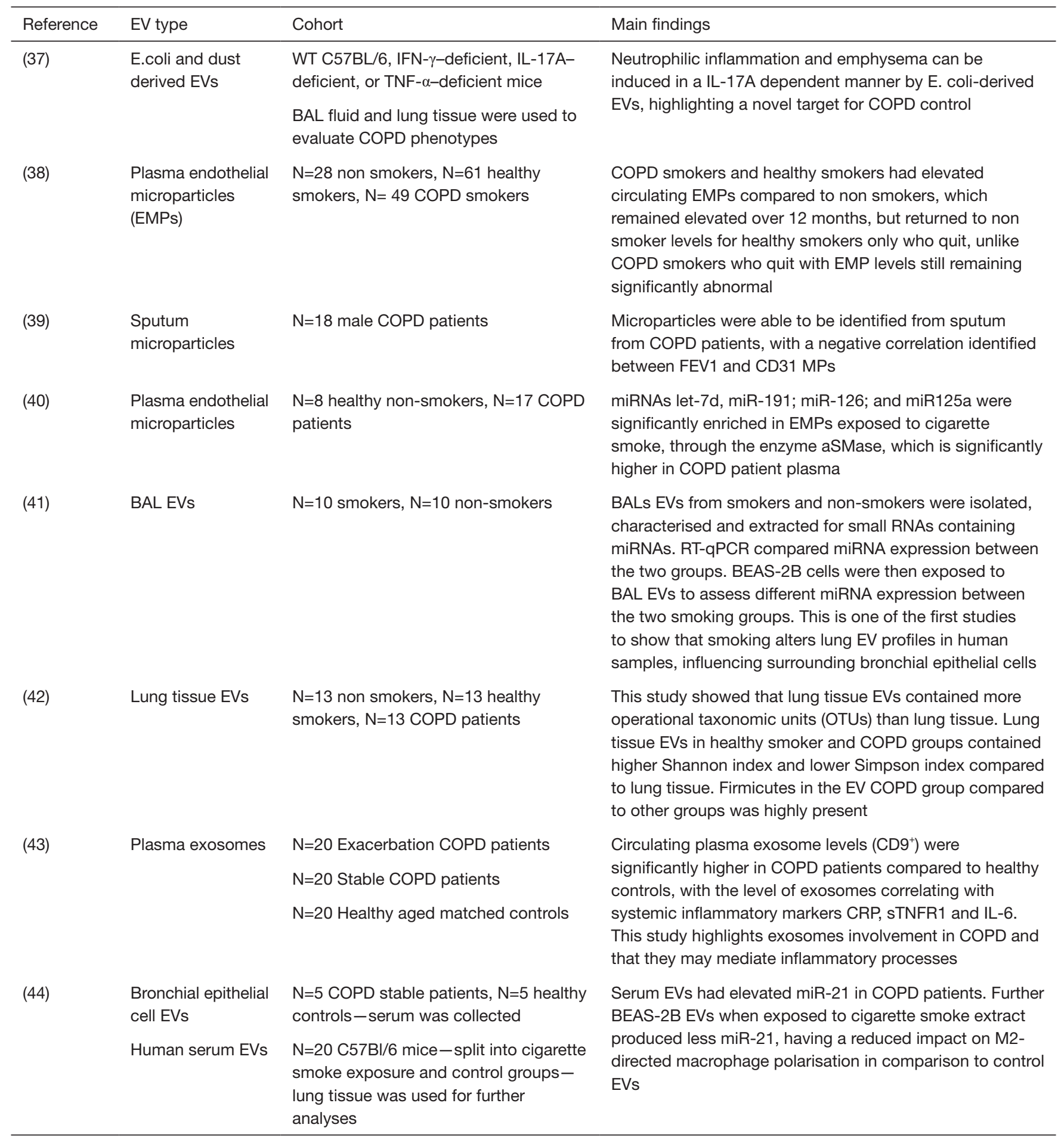

EV, extracellular vesicle; COPD, chronic obstructive pulmonary disease. 
Viruses exploit EV machinery to uptake virus-expressed molecules (such as virions, proteins, mRNAs and miRNAs) in exosomes $(50,58)$, which can then be transferred to uninfected recipient cells through a number of mechanisms, including interaction with the ESCRT pathway, as well as viruses utilising the Ras-related protein Rab pathway (50).

Recently EV based vaccines using pathogen EVs and their bioactive cargo have shown promising results (59-61). Clinical trials assessing OMVs have been advantageous compared to conventional vaccines against infectious diseases, as unlike cell and virus therapies, EVs cannot multiple or divide, suggesting they are therefore safer and less tumorigenic and infectious (62). Risks such as pathogen co-isolation with EVs has been considered, which is particularly relevant for viruses, who have similar biophysical properties to EVs (63). Overall EV-based vaccines used as antimicrobial treatment in preclinical and clinical trials has so far been reported to be well-tolerated and feasible, however further validation in humans is needed to show consistent immunostimulatory therapeutic effect (63).

\section{Circulating miRNAs and EV miRNAs as potential COPD biomarkers}

Biomarkers are clinical features that reflect disease activity and fluctuate with disease state, making them useful for diagnosis, monitoring of disease progression, as well as response to therapy $(64,65)$. EVs have been identified as novel disease biomarkers due to their ability to reflect their parent cells physiological state and microenvironment, as well as being highly stable in circulating bodily fluids, due to their phospholipid bilayer, and have the ability to package an array of disease associated biomolecules, overall making them strong biomarker candidates.

microRNAs (miRNAs) are short non-coding RNAs that regulate gene expression post-transcriptionally (66). miRNAs have been shown to be stable in a variety of body fluids, including saliva, sputum, urine, breast milk and blood (66). Specific miRNAs are have been demonstrated to be selectively exported into EVs, while others are excluded (67). The mechanisms behind this are complex but studies have demonstrated miRNA sorting into EVs through the RISC complex loading miRNAs into the multivesicular bodies (68), sumoylated heterogenous nuclear ribonucleoprotein A2B1 (69) and Annexin A2 (70). Given the transportability of vesicles, the role of which miRNAs are that packaged into EVs during different disease states is gaining increasing attention. Based on recent studies,
miRNAs are differentially enriched in EVs, and in respect to different lung diseases, their expression can change, further altering recipient cells biological processes and overall disease pathophysiology (71).

A number of studies have reported various circulating miRNAs that are involved in the development and progression of COPD, including emphysema severity (72) [lists of studied circulating miRNAs have been extensively detailed in previous reviews $(73,74)]$.

Recent studies investigating exosomal miRNA expression include a study by Fujita el al, who reported that intercellular crosstalk and COPD pathogenesis were modulated by miRNAs through EV-mediated miRNA transfer (36). Specifically, miR-210 expression was significantly increased in bronchial epithelial cell (BEC)derived EVs as well as BECs after cigarette smoke exposure, promoting airway fibrosis in COPD pathogenesis (36). Another study investigating EV transfer of miRNAs in COPD pathogenesis reported microparticles were secreted on the ceramide-synthesis enzyme sphingomyelinase (aSMase), which induced the release of select miRNAs including miR-191, miR-126 and miR-125a, to be transferred to macrophages, promoting the clearance of apoptotic cells (40). Exosomal miR-21 derived from bronchial epithelial cells has also recently been found to be responsible for myofibroblast differentiation in response to cigarette smoke, with exosomal miR-21 levels found to be high in the sera of smokers and COPD patients (75).

Due to the differential changes in plasma mRNAs, exosomal miRNAs are therefore not equivalent to the measurement of circulating miRNAs (76). Therefore future studies looking into exosomal miRNAs can be useful to extend previous reports of circulating miRNAs with biomarker potential, for example, by identifying if both miRNA levels of plasma and plasma exosomes are similar.

\section{Therapeutic potential of EVs in COPD}

\section{EVs as cargo delivery vehicles}

The utility of EVs, in particular exosomes as a diagnostic marker for diseases and potential for therapeutic intervention, is currently under intense investigation. One of the first considerations for developing an EV therapeutic is the cellular source. Mesenchymal stromal cells (MSCs) have been reported to be anti-inflammatory and regenerative and pre-clinical trials have been undertaken using MSCs for the treatment of COPD (77-79). However 
there is still no evidence for clinically relevant effects and further study of MSC-based treatments for COPD patients is needed (79). Efforts have increased in the development of MSC-EVs as a potential therapeutic, with studies reporting that the immune-regulatory function and regenerative capacity of MSCs is mimicked in their EVs (80-82). Issues such as culture conditions, yield, manufacturability and working with mammalian cell EVs themselves, have been addressed by research groups who have reported using EVs derived from fruits and vegetables, as they are able to be loaded with small molecular cargo, which can mediate a therapeutic effect in animal models (83-85). Another consideration for $\mathrm{EV}$ therapeutics is the route of administration for use in patients (86).

EVs can be modified for therapeutic use through molecular engineering techniques. Two basic approaches can be utilized to modify EVs for therapeutics: (I) exogenously, which involves incorporating therapeutic cargo (small molecules/proteins or RNAs) into or onto isolated $\mathrm{EVs}$, or (II) endogenously, which involves providing cells with the means to incorporate therapeutic cargo into EVs during their biogenesis (63). Exogenous methods to package therapeutic cargo into EVs occurs after EV collection by co-incubation, electroporation and sonication (87-89). This has proved successful in studies that incubated EVs with curcumin, which improved anti-inflammatory and bioavailability of this drug in an inflammation mouse model (90). Endogenous methods for loading therapeutic cargo involve genetically modifying parental cells to over express target RNA or protein of interest, which is then naturally taken up and secreted by EVs (63). Similar methods can be used as exogenous EV loading such as incubation, transfection and or transduction (91-93). A novel study by Alvarez-Erviti et al. delivered siRNAs targeting housekeeping genes and a therapeutic target for Alzheimer's disease using exosomes into mouse brains, using electroporation as a method of transfection (88). Another study by Zhang et al. used a novel protocol to manipulate exosomal miRNAs with high efficiency and successfully deliver them to recipient cells (94). Through the modification of a calcium chloride transfection method, miRNA mimics and inhibitors were enhanced, further allowing the direct transfection of exosomes instead of transfection of mother cells (94).

A number of studies that have investigated the role that EVs play in disease pathogenesis has shed light on their use to deliver cell specific therapy as cargo $(95,96)$. It has been reported that for exosome loading, miRNAs are not randomly selected, with exosomes enriched in 3'UTR RNA fragments being more likely to be sorted into exosomes $(69,97)$. EVs protect miRNAs from digestion and degradation through shuttling and evasion of immune system surveillance due to EVs carrying the same surface proteins as their cell of origin (98). This further highlights the utility of EVs as a mediated small nucleotide delivery system (94).

In order for $\mathrm{EV}$-mediated miRNA delivery to be used as a treatment therapy, several issues still need to be addressed. This includes overcoming miRNA enrichment into EVs, as well as assessing EV shuttling miRNAs physiological and pathological effects on recipient cells (94). Studies have reported that the load of miRNAs from tumour cells are low in individual exosomes from patient plasma, and that host-derived exosomes are unable to deliver a sufficient copies of desired miRNAs without their composition being manipulated (99). However one of the benefits of using host/patient serum circulating exosomes is the high cell specificity and less likely chance to cause a reaction or rejection by the recipient (94).

Another additional consideration is the use of EVs to transfer bioactive cargo targeting gene replacement or inhibiting expression of inherited diseases (63). Adenoassociated vectors (AAVs) are a novel gene therapy tool that has shown promising results in clinical trials (100). Further a study by Maguire et al. was able to overcome limitations associated with using AAVs through the use of their associated EVs (exo-AAVs), which were found to be superior to AAVs, in neutralising anti AAV antibodies in vitro and in vivo, as well as transduction efficiency (101). The use of exo-AAV as a novel gene delivery system may holds clinical potential for the treatment of genetic disorder alpha-1 antitrypsin deficiency, which can lead to emphysema and COPD.

\section{Therapeutic potential for probiotic EVs}

Probiotics are defined as "live organisms, which when applied in adequate amounts confer a health benefit to the host" (102). Probiotic Lactobacillus is a Grampositive facultative anaerobic bacteria and considered non-pathogenic $(103,104)$, with reported benefits such as inhibiting cytokine-induced apoptosis, as well as decreasing the pathogenicity of other pathogens such as E.coli $(105,106)$.

EVs are able to influence their host through a variety of cellular responses, which proves essential for bacteria to communicate with host cells as a layer of mucin prevents 
physical contact between host tissue and bacteria $(107,108)$. EVs also have the potential to mediate therapeutic molecule delivery without inducing adverse immune reactions (107). Therefore the EVs produced by probiotic bacteria may exert a positive effect in stabilising dysbiosis in the lung microenvironment, further increasing treatment efficacy.

Studies investigating the bacterial microbiome in lung tissue have reported that the bacteria Lactobacillus is unique to severe COPD patients (GOLD stage 4) in comparison to smoking and non-smoking control groups (109). A study by Sze et al. reported that the loss of GD1 (glycerol dehydratase gene), most commonly found on plasmids with Lactobacillus reuteri, results in increasing COPD severity and that GD1 holds anti-inflammatory properties, highlighting Lactobacillus potential as a possible therapeutic (110).

Further, work investigating probiotic conditioning of $C$. elegans with Lactobacillus derived EVs reported protection against VRE (vancomycin-resistant enterococci, an antibiotic resistant bacterium) infection, highlighting that EVs produced by this bacterium up regulate the expression of host defence genes and exert a protective effect on the host. This study supports that the use of probiotic-derived $\mathrm{EVs}$, and not just the probiotic bacteria, is a novel therapy to treat antimicrobial-resistant pathogens (111).

\section{Future directions}

The clinical efficacy of EV cargo transfer for treating disease has previously been undertaken in phase 1 and 2 trials for a number of diseases [as previously described in detail (63)]. For non-small cell lung cancer (NSCLC), tumour antigen-loaded DC-derived exosomes were found to be well-tolerated by patients and safe for treatment, boosting antitumor immunity (112). Another study using MSC-derived exosome therapy has been found to reduce symptoms of steroid-refractory graft $v s$. host disease with no noticeable side effects (113).

Overall issues that still need to be addressed for future implementation of EVs as a diagnostic or therapeutic tool into clinic are a more standardised protocol for $\mathrm{EV}$ isolation, particularly which population of $\mathrm{EVs}$ is being investigated, and if the bioactive cargo specific to disease status is directly being identified from this specific EV population and not just extracellular content. Additional considerations include the mechanisms behind EV bioactive cargo incorporation and packaging, as well as the mechanisms behind recipient cells uptake EVs and their bioactive cargo.

\section{Conclusions}

COPD is a heterogeneous disease, characterised by airway inflammation, destruction of lung tissue and airflow limitation associated with airway remodelling. Exacerbations are a major cause of disease progression, morbidity and mortality and given the lack of curative treatments, new diagnostic and therapeutics for COPD are highly relevant. EVs are highly stable and potent packages of cellular information, and are able to exchange their bioactive cargo to recipient cells around the body, influencing physiological and pathological conditions. Furthermore EVs have significant therapeutic potential due to their ability to be manipulated and engineered to be used as targeted diagnostic and therapeutic tools. This review has highlighted recent evidence of the potential of EVs as biomarkers, and how this could be applied specifically for COPD management. Future studies investigating bioengineered EVs and their use as novel drug delivery vehicles hold great promise for therapeutic translation into the clinic.

\section{Acknowledgments}

Funding: The Prince Charles Hospital Foundation (HO, IY), NHMRC project grant APP1121740 (IY), The University of Queensland PhD scholarship (HO).

\section{Footnote}

Conflicts of Interest: The authors have no conflicts of interest to declare.

Ethical Statement: The authors are accountable for all aspects of the work in ensuring that questions related to the accuracy or integrity of any part of the work are appropriately investigated and resolved.

\section{References}

1. Singh D, Agusti A, Anzueto A, et al. Global Strategy for the Diagnosis, Management, and Prevention of Chronic Obstructive Lung Disease: the GOLD science committee report 2019. Eur Respir J 2019;53. doi: 10.1183/13993003.00164-2019.

2. Vogelmeier CF, Criner GJ, Martinez FJ, et al. Global Strategy for the Diagnosis, Management, and Prevention of Chronic Obstructive Lung Disease 2017 Report. 
GOLD Executive Summary. Am J Respir Crit Care Med 2017;195:557-82.

3. GOLD. Global Strategy for the Diagnosis, Management, and Prevention of Chronic Obstructive Pulmonary Disease - 2018 Report. 2018. Available online: http://goldcopd. org/wp-content/uploads/2017/11/GOLD-2018-v6.0FINAL-revised-20-Nov_WMS.pdf

4. Australia TLF. Economic impact of COPD. 2008. Available online: http://lungfoundation.com.au/healthprofessionals/clinical-resources/publications/economicimpact-of-copd/

5. WHO. COPD predicted to be thrid leading cause of death in 2030. 2008. Available online: http://www.who.int/ respiratory/copd/World_Health_Statistics_2008/en/

6. 2019 Global Initiative for Chronic Obstructive Lung Disease I. Global Initiative for Chronic Obstructive Lung Disease. 2019. Available online: https://goldcopd.org/ wp-content/uploads/2018/11/GOLD-2019-POCKETGUIDE-FINAL_WMS.pdf

7. Hurst JR, Vestbo J, Anzueto A, et al. Susceptibility to exacerbation in chronic obstructive pulmonary disease. $\mathrm{N}$ Engl J Med 2010;363:1128-38.

8. Fujita Y, Kosaka N, Araya J, et al. Extracellular vesicles in lung microenvironment and pathogenesis. Trends Mol Med 2015;21:533-42.

9. Benedikter BJ, Wouters EFM, Savelkoul PHM, et al. Extracellular vesicles released in response to respiratory exposures: implications for chronic disease. J Toxicol Environ Health B Crit Rev 2018;21:142-60.

10. Akers JC, Gonda D, Kim R, et al. Biogenesis of extracellular vesicles (EV): exosomes, microvesicles, retrovirus-like vesicles, and apoptotic bodies. J Neurooncol 2013;113:1-11.

11. EL Andaloussi S, Mäger I, Breakefield XO, et al. Extracellular vesicles: biology and emerging therapeutic opportunities. Nature Reviews Drug Discovery 2013;12:347.

12. Cocucci E, Racchetti G, Meldolesi J. Shedding microvesicles: artefacts no more. Trends Cell Biol 2009; 19:43-51.

13. Kinoshita T, Yip KW, Spence T, et al. MicroRNAs in extracellular vesicles: potential cancer biomarkers. J Hum Genet 2017;62:67.

14. Hessvik NP, Llorente A. Current knowledge on exosome biogenesis and release. Cell Mol Life Sci 2018;75:193-208.

15. Muralidharan-Chari V, Clancy JW, Sedgwick A, et al. Microvesicles: mediators of extracellular communication during cancer progression. J Cell Sci 2010;123:1603-11.
16. Bebelman MP, Smit MJ, Pegtel DM, et al. Biogenesis and function of extracellular vesicles in cancer. Pharmacol Ther 2018;188:1-11.

17. Frydrychowicz M, Kolecka-Bednarczyk A, Madejczyk M, et al. Exosomes - structure, biogenesis and biological role in non-small-cell lung cancer. Scand J Immunol 2015;81:2-10.

18. Thery C, Amigorena S, Raposo G, et al. Isolation and characterization of exosomes from cell culture supernatants and biological fluids. Curr Protoc Cell Biol 2006; Chapter 3:Unit 322.

19. Tauro BJ, Greening DW, Mathias RA, et al. Comparison of ultracentrifugation, density gradient separation, and immunoaffinity capture methods for isolating human colon cancer cell line LIM1863-derived exosomes. Methods 2012;56:293-304.

20. Taylor DD, Shah S. Methods of isolating extracellular vesicles impact down-stream analyses of their cargoes. Methods 2015;87:3-10.

21. Brownlee Z, Lynn KD, Thorpe PE, et al. A novel "salting-out" procedure for the isolation of tumor-derived exosomes. J Immunol Methods 2014;407:120-6.

22. Gámez-Valero A, Monguió-Tortajada M, Carreras-Planella L, et al. Size-Exclusion Chromatography-based isolation minimally alters Extracellular Vesicles' characteristics compared to precipitating agents. Sci Rep 2016;6:33641.

23. Deregibus MC, Figliolini F, D'Antico S, et al. Chargebased precipitation of extracellular vesicles. Int J Mol Med 2016;38:1359-66.

24. Konoshenko MY, Lekchnov EA, Vlassov AV, et al. Isolation of Extracellular Vesicles: General Methodologies and Latest Trends. Biomed Res Int 2018;2018:8545347.

25. Lötvall J, Hill AF, Hochberg F, et al. Minimal experimental requirements for definition of extracellular vesicles and their functions: a position statement from the International Society for Extracellular Vesicles. J Extracell Vesicles 2014;3:26913.

26. Witwer KW, Soekmadji C, Hill AF, et al. Updating the MISEV minimal requirements for extracellular vesicle studies: building bridges to reproducibility. J Extracell Vesicles 2017;6:1396823.

27. Shah R, Patel T, Freedman JE. Circulating Extracellular Vesicles in Human Disease. N Engl J Med 2018;379:958-66.

28. Gordon C, Gudi K, Krause A, et al. Circulating endothelial microparticles as a measure of early lung destruction in cigarette smokers. Am J Respir Crit Care Med 2011;184:224-32. 
29. Takahashi T, Kobayashi S, Fujino N, et al. Increased circulating endothelial microparticles in COPD patients: a potential biomarker for COPD exacerbation susceptibility. Thorax 2012;67:1067.

30. Li CJ, Liu Y, Chen Y, et al. Novel proteolytic microvesicles released from human macrophages after exposure to tobacco smoke. Am J Pathol 2013;182:1552-62.

31. Thomashow MA, Shimbo D, Parikh MA, et al. Endothelial microparticles in mild chronic obstructive pulmonary disease and emphysema. The Multi-Ethnic Study of Atherosclerosis Chronic Obstructive Pulmonary Disease study. Am J Respir Crit Care Med 2013;188:60-8.

32. Cordazzo C, Petrini S, Neri T, et al. Rapid shedding of proinflammatory microparticles by human mononuclear cells exposed to cigarette smoke is dependent on $\mathrm{Ca} 2+$ mobilization. Inflamm Res 2014;63:539-47.

33. Eltom S, Dale N, Raemdonck KR, et al. Respiratory infections cause the release of extracellular vesicles: implications in exacerbation of asthma/COPD. PLoS One 2014;9:e101087.

34. Liu H, Ding L, Zhang Y, et al. Circulating endothelial microparticles involved in lung function decline in a rat exposed in cigarette smoke maybe from apoptotic pulmonary capillary endothelial cells. J Thorac Dis 2014;6:649-55.

35. Moon HG, Kim SH, Gao J, et al. CCN1 secretion and cleavage regulate the lung epithelial cell functions after cigarette smoke. Am J Physiol Lung Cell Mol Physiol 2014;307:L326-37.

36. Fujita Y, Araya J, Ito S, et al. Suppression of autophagy by extracellular vesicles promotes myofibroblast differentiation in COPD pathogenesis. J Extracell Vesicles 2015;4:28388.

37. Kim YS, Lee WH, Choi EJ, et al. Extracellular vesicles derived from Gram-negative bacteria, such as Escherichia coli, induce emphysema mainly via IL-17A-mediated neutrophilic inflammation. J Immunol 2015;194:3361-8.

38. Strulovici-Barel Y, Staudt MR, Krause A, et al. Persistence of circulating endothelial microparticles in COPD despite smoking cessation. Thorax 2016;71:1137-44.

39. Lacedonia D, Carpagnano GE, Trotta T, et al. Microparticles in sputum of COPD patients: a potential biomarker of the disease? Int J Chron Obstruct Pulmon Dis 2016;11:527-33.

40. Serban KA, Rezania S, Petrusca DN, et al. Structural and functional characterization of endothelial microparticles released by cigarette smoke. Sci Rep 2016;6:31596.

41. Héliot A, Landkocz Y, Roy Saint-Georges F, et al.
Smoker extracellular vesicles influence status of human bronchial epithelial cells. Int J Hyg Environ Health 2017;220:445-54.

42. Kim HJ, Kim YS, Kim KH, et al. The microbiome of the lung and its extracellular vesicles in nonsmokers, healthy smokers and COPD patients. Exp Mol Med 2017;49:e316.

43. Tan DBA, Armitage J, Teo TH, et al. Elevated levels of circulating exosome in COPD patients are associated with systemic inflammation. Respir Med 2017;132:261-4.

44. He S, Chen D, Hu M, et al. Bronchial epithelial cell extracellular vesicles ameliorate epithelial-mesenchymal transition in COPD pathogenesis by alleviating M2 macrophage polarization. Nanomedicine 2019;18:259-71.

45. Kulshreshtha A, Ahmad T, Agrawal A, et al. Proinflammatory role of epithelial cell-derived exosomes in allergic airway inflammation. J Allergy Clin Immunol 2013;131:1194-203, 1203.e1-14.

46. Anand PK, Anand E, Bleck CKE, et al. Exosomal Hsp70 Induces a Pro-Inflammatory Response to Foreign Particles Including Mycobacteria. PLoS One 2010;5:e10136.

47. Fujita Y, Kadota T, Araya J, et al. Extracellular Vesicles: New Players in Lung Immunity. Am J Respir Cell Mol Biol 2018;58:560-5.

48. Genschmer KR, Russell DW, Lal C, et al. Activated PMN Exosomes: Pathogenic Entities Causing Matrix Destruction and Disease in the Lung. Cell 2019;176:11326.e15.

49. Sethi S, Murphy TF. Infection in the pathogenesis and course of chronic obstructive pulmonary disease. N Engl J Med 2008;359:2355-65.

50. Rodrigues M, Fan J, Lyon C, et al. Role of Extracellular Vesicles in Viral and Bacterial Infections: Pathogenesis, Diagnostics, and Therapeutics. Theranostics 2018;8:2709-21.

51. Raab-Traub N, Dittmer DP. Viral effects on the content and function of extracellular vesicles. Nat Rev Microbiol 2017;15:559-72.

52. Kim JH, Lee J, Park J, et al. Gram-negative and Grampositive bacterial extracellular vesicles. Semin Cell Dev Biol 2015;40:97-104.

53. Lee EY, Choi DY, Kim DK, et al. Gram-positive bacteria produce membrane vesicles: Proteomics-based characterization of Staphylococcus aureus-derived membrane vesicles. Proteomics 2009;9:5425-36.

54. Kim MR, Hong SW, Choi EB, et al. Staphylococcus aureus-derived extracellular vesicles induce neutrophilic pulmonary inflammation via both Th1 and Th17 cell responses. Allergy 2012;67:1271-81. 
55. Kim YS, Choi EJ, Lee WH, et al. Extracellular vesicles, especially derived from Gram-negative bacteria, in indoor dust induce neutrophilic pulmonary inflammation associated with both Th1 and Th17 cell responses. Clin Exp Allergy 2013;43:443-54.

56. Altan-Bonnet N. Extracellular vesicles are the Trojan horses of viral infection. Curr Opin Microbiol 2016;32:77-81.

57. Hewitt R, Farne H, Ritchie A, et al. The role of viral infections in exacerbations of chronic obstructive pulmonary disease and asthma. Ther Adv Respir Dis 2016;10:158-74.

58. Mori Y, Koike M, Moriishi E, et al. Human herpesvirus-6 induces MVB formation, and virus egress occurs by an exosomal release pathway. Traffic 2008;9:1728-42.

59. Bhatnagar S, Shinagawa K, Castellino FJ, et al. Exosomes released from macrophages infected with intracellular pathogens stimulate a proinflammatory response in vitro and in vivo. Blood 2007;110:3234-44.

60. Aline F, Bout D, Amigorena S, et al. Toxoplasma gondii antigen-pulsed-dendritic cell-derived exosomes induce a protective immune response against $\mathrm{T}$. gondii infection. Infect Immun 2004;72:4127-37.

61. Roy K, Hamilton DJ, Munson GP, et al. Outer membrane vesicles induce immune responses to virulence proteins and protect against colonization by enterotoxigenic Escherichia coli. Clin Vaccine Immunol 2011;18:1803-8.

62. Kaparakis-Liaskos M, Ferrero RL. Immune modulation by bacterial outer membrane vesicles. Nat Rev Immunol 2015;15:375-87.

63. Wiklander OPB, Brennan MÁ, Lötvall J, et al. Advances in therapeutic applications of extracellular vesicles. Sci Transl Med 2019;11:eaav8521.

64. Chen YW, Leung JM, Sin DD. A Systematic Review of Diagnostic Biomarkers of COPD Exacerbation. PLoS One 2016;11:e0158843.

65. Shaw JG, Vaughan A, Dent AG, et al. Biomarkers of progression of chronic obstructive pulmonary disease (COPD). J Thorac Dis 2014;6:1532-47.

66. Zhang J, Li S, Li L, et al. Exosome and Exosomal MicroRNA: Trafficking, Sorting, and Function. Genomics Proteomics Bioinformatics 2015;13:17-24.

67. Nolte-'t Hoen EN, Buermans HP, Waasdorp M, et al. Deep sequencing of RNA from immune cell-derived vesicles uncovers the selective incorporation of small noncoding RNA biotypes with potential regulatory functions. Nucleic Acids Res 2012;40:9272-85.

68. Gibbings DJ, Ciaudo C, Erhardt M, et al. Multivesicular bodies associate with components of miRNA effector complexes and modulate miRNA activity. Nat Cell Biol 2009;11:1143-9.

69. Villarroya-Beltri C, Gutierrez-Vazquez C, Sanchez-Cabo F, et al. Sumoylated hnRNPA2B1 controls the sorting of miRNAs into exosomes through binding to specific motifs. Nat Commun 2013;4:2980.

70. Hagiwara K, Katsuda T, Gailhouste L, et al. Commitment of Annexin A2 in recruitment of microRNAs into extracellular vesicles. FEBS Lett 2015;589:4071-8.

71. Pattarayan D, Thimmulappa RK, Ravikumar V, et al. Diagnostic Potential of Extracellular MicroRNA in Respiratory Diseases. Clin Rev Allergy Immunol 2018;54:480-92.

72. Savarimuthu Francis SM, Davidson MR, Tan ME, et al. MicroRNA-34c is associated with emphysema severity and modulates SERPINE1 expression. BMC Genomics 2014;15:88-.

73. De Smet EG, Mestdagh P, Vandesompele J, et al. Noncoding RNAs in the pathogenesis of COPD. Thorax 2015;70:782.

74. Salimian J, Mirzaei H, Moridikia A, et al. Chronic obstructive pulmonary disease: MicroRNAs and exosomes as new diagnostic and therapeutic biomarkers. J Res Med Sci 2018;23:27.

75. Xu H, Ling M, Xue J, et al. Exosomal microRNA-21 derived from bronchial epithelial cells is involved in aberrant epithelium-fibroblast cross-talk in COPD induced by cigarette smoking. Theranostics 2018;8:5419-33.

76. Xie JX, Fan X, Drummond CA, et al. MicroRNA profiling in kidney disease: Plasma versus plasma-derived exosomes. Gene 2017;627:1-8.

77. Weiss DJ, Casaburi R, Flannery R, et al. A placebocontrolled, randomized trial of mesenchymal stem cells in COPD. Chest 2013;143:1590-8.

78. Stolk J, Broekman W, Mauad T, et al. A phase I study for intravenous autologous mesenchymal stromal cell administration to patients with severe emphysema. QJM 2016;109:331-6.

79. Broekman W, Khedoe PPSJ, Schepers K, et al. Mesenchymal stromal cells: a novel therapy for the treatment of chronic obstructive pulmonary disease? Thorax 2018;73:565.

80. Lee C, Mitsialis SA, Aslam M, et al. Exosomes mediate the cytoprotective action of mesenchymal stromal cells on hypoxia-induced pulmonary hypertension. Circulation 2012;126:2601-11. 
81. Ahn SY, Park WS, Kim YE, et al. Vascular endothelial growth factor mediates the therapeutic efficacy of mesenchymal stem cell-derived extracellular vesicles against neonatal hyperoxic lung injury. Exp Mol Med 2018;50:26.

82. Khatri M, Richardson LA, Meulia T. Mesenchymal stem cell-derived extracellular vesicles attenuate influenza virusinduced acute lung injury in a pig model. Stem Cell Res Ther 2018;9:17.

83. Raimondo S, Naselli F, Fontana S, et al. Citrus limonderived nanovesicles inhibit cancer cell proliferation and suppress CML xenograft growth by inducing TRAILmediated cell death. Oncotarget 2015;6:19514-27.

84. Mu J, Zhuang X, Wang Q, et al. Interspecies communication between plant and mouse gut host cells through edible plant derived exosome-like nanoparticles. Mol Nutr Food Res 2014;58:1561-73.

85. Wang B, Zhuang X, Deng ZB, et al. Targeted drug delivery to intestinal macrophages by bioactive nanovesicles released from grapefruit. Mol Ther 2014;22:522-34.

86. Wiklander OPB, Nordin JZ, O'Loughlin A, et al. Extracellular vesicle in vivo biodistribution is determined by cell source, route of administration and targeting. J Extracell Vesicles 2015;4:26316.

87. Didiot MC, Hall LM, Coles AH, et al. Exosome-mediated Delivery of Hydrophobically Modified siRNA for Huntingtin mRNA Silencing. Mol Ther 2016;24:1836-47.

88. Alvarez-Erviti L, Seow Y, Yin H, et al. Delivery of siRNA to the mouse brain by systemic injection of targeted exosomes. Nat Biotechnol 2011;29:341-5.

89. Lamichhane TN, Jeyaram A, Patel DB, et al. Oncogene Knockdown via Active Loading of Small RNAs into Extracellular Vesicles by Sonication. Cell Mol Bioeng 2016;9:315-24.

90. Sun D, Zhuang X, Xiang X, et al. A novel nanoparticle drug delivery system: the anti-inflammatory activity of curcumin is enhanced when encapsulated in exosomes. Mol Ther 2010;18:1606-14.

91. Pascucci L, Cocce V, Bonomi A, et al. Paclitaxel is incorporated by mesenchymal stromal cells and released in exosomes that inhibit in vitro tumor growth: a new approach for drug delivery. J Control Release 2014;192:262-70.

92. Ohno S, Takanashi M, Sudo K, et al. Systemically injected exosomes targeted to EGFR deliver antitumor microRNA to breast cancer cells. Mol Ther 2013;21:185-91.

93. Zhang Y, Liu D, Chen X, et al. Secreted monocytic miR150 enhances targeted endothelial cell migration. Mol Cell
2010;39:133-44.

94. Zhang D, Lee H, Zhu Z, et al. Enrichment of selective miRNAs in exosomes and delivery of exosomal miRNAs in vitro and in vivo. Am J Physiol Lung Cell Mol Physiol 2017;312:L110-21.

95. Minciacchi VR, Freeman MR, Di Vizio D. Extracellular vesicles in cancer: exosomes, microvesicles and the emerging role of large oncosomes. Semin Cell Dev Biol 2015;40:41-51.

96. Tickner JA, Urquhart AJ, Stephenson SA, et al. Functions and therapeutic roles of exosomes in cancer. Front Oncol 2014;4:127.

97. Batagov AO, Kurochkin IV. Exosomes secreted by human cells transport largely mRNA fragments that are enriched in the 3'-untranslated regions. Biol Direct 2013;8:12.

98. Etheridge A, Lee I, Hood L, et al. Extracellular microRNA: a new source of biomarkers. Mutat Res 2011;717:85-90.

99. Chevillet JR, Kang Q, Ruf IK, et al. Quantitative and stoichiometric analysis of the microRNA content of exosomes. Proc Natl Acad Sci U S A 2014;111:14888-93.

100. Grimm D, Buning H. Small But Increasingly Mighty: Latest Advances in AAV Vector Research, Design, and Evolution. Hum Gene Ther 2017;28:1075-86.

101. Maguire CA, Balaj L, Sivaraman S, et al. Microvesicleassociated AAV vector as a novel gene delivery system. Mol Ther 2012;20:960-71.

102. Mortaz E, Adcock IM, Folkerts G, et al. Probiotics in the management of lung diseases. Mediators Inflamm 2013;2013:751068-.

103. Stiles ME, Holzapfel WH. Lactic acid bacteria of foods and their current taxonomy. Int J Food Microbiol 1997;36:1-29.

104. Makarova K, Slesarev A, Wolf Y, et al. Comparative genomics of the lactic acid bacteria. Proc Natl Acad Sci U S A 2006;103:15611-6.

105. Yan F, Polk DB. Probiotic bacterium prevents cytokineinduced apoptosis in intestinal epithelial cells. J Biol Chem 2002;277:50959-65.

106. Medellin-Peña MJ, Griffiths MW. Effect of molecules secreted by Lactobacillus acidophilus strain La-5 on Escherichia coli O157:H7 colonization. Appl Environ Microbiol 2009;75:1165-72.

107. Yáñez-Mó M, Siljander PR, Andreu Z, et al. Biological properties of extracellular vesicles and their physiological functions. J Extracell Vesicles 2015;4:27066.

108. Kuehn MJ, Kesty NC. Bacterial outer membrane vesicles and the host-pathogen interaction. Genes Dev 
2005;19:2645-55.

109. Sze MA, Dimitriu PA, Hayashi S, et al. The lung tissue microbiome in chronic obstructive pulmonary disease. Am J Respir Crit Care Med 2012;185:1073-80.

110.Sze MA, Utokaparch S, Elliott WM, et al. Loss of GD1positive Lactobacillus correlates with inflammation in human lungs with COPD. BMJ Open 2015;5:e006677.

111.Li M, Lee K, Hsu M, et al. Lactobacillus-derived extracellular vesicles enhance host immune responses

Cite this article as: O'Farrell HE, Yang IA. Extracellular vesicles in chronic obstructive pulmonary disease (COPD). J Thorac Dis 2019;11(Suppl 17):S2141-S2154. doi: 10.21037/ jtd.2019.10.16 against vancomycin-resistant enterococci. BMC Microbiol 2017;17:66-.

112. Morse MA, Garst J, Osada T, et al. A phase I study of dexosome immunotherapy in patients with advanced nonsmall cell lung cancer. J Transl Med 2005;3:9.

113.Kordelas L, Rebmann V, Ludwig AK, et al. MSC-derived exosomes: a novel tool to treat therapy-refractory graftversus-host disease. Leukemia 2014;28:970-3. 